\title{
The Study of Control Strategy for Bolt Tightening Robot on Power Transmission Lines
}

\author{
Liqiang $\mathrm{ZHONG}^{1 *}$ \\ ${ }^{1}$ Electric Power Research Institute of Guangdong Power \\ Grid Co, Ltd. \\ Guangzhou, Guangdong 510080, China \\ Email: liqiangzhong@hotmail.com \\ +* Corresponding author \\ Ruoyun $\mathrm{LI}^{2}$ \\ ${ }^{2}$ College of Electric and Information Engineering \\ Changsha University of Science and Technology \\ Changsha, China \\ E-mail: fss508@163.com
}

\author{
Shaosheng FAN ${ }^{2}$ \\ ${ }^{2}$ College of Electric and Information Engineering \\ Changsha University of Science and Technology \\ Changsha, China \\ E-mail: fss508@163.com
}

\author{
Di YANG ${ }^{2}$ \\ ${ }^{2}$ College of Electric and Information Engineering \\ Changsha University of Science and Technology \\ Changsha, China \\ E-mail: fss508@163.com
}

\begin{abstract}
A new control strategy and its real-time implementation were presented for a power transmission line bolt tightening robot. In order to overcome the influence of the wind, a prediction algorithm of multi-sensor information fusion optimal Kalman filter is adopted to predict the trajectory of the camera. The trajectory of the bolt uses a least squares method based on the interest area. Torque coefficient control method was presented to assure the conformity of the pre-tightening force of different screw threads. Experimental results show that the proposed bolt aligning and tightening strategy can achieve the work timely and have high efficiency.
\end{abstract}

Keywords-sensor fusion; kalman filter; least square method; interest area; infrared detection; torque coefficient control

\section{INTRODUCTION}

Bolt-type power connectors are widely used on power transmission lines due to their simple structure and low cost[1]. However, the outdoor working condition for bolts on power transmission lines is rather complicated. The vagaries of climate will cause vibration of the power transmission lines. Due to the vibration, bolts on the lines will be loosened which may cause severe damage to the power transmission lines, bringing great financial ruin to the power suppliers and the users. The continuous maintenance of the power transmission lines, especially the bolts tightening task, is the assurance for the stable and secure operation of power system. Considering lowering the risk of maintenance work on the lines for manual operation, research on robot based power-line inspection and maintenance becomes more and more important.

Lots of high voltage electric power bolt tightening robots have been designed. A bolt tightening robot for power transmission line is proposed in [2]-[3].A semiautonomous robot system that performs tasks using tasklevel instructions is proposed in [4].These robots are manual remote-controlled, since people are far away from the operating scene, it is difficult for operators to pinpoint the spanner at the bolt, i.e. it is inefficient for these kinds of robots because the instructions from people are not always reliable. They also just based on experience to tightening the bolt which may cause severe damage to the power transmission lines. The wind will cause vibration of the robot and the bolt result in the alignment of bolt become difficult. The inner stress of the bolt will change under the influence of the environment, so the control torque should be changed. To solve these problems, a bolt tightening robot automatically search and tighten is proposed.

In this paper, a bolt tightening robot and its corresponding bolt tightening strategy is proposed to provide a novel mechanism for bolts tightening task. Considering the influence of the wind, the motion of the robot and the bolt are nonlinear, and their vibration frequency is different, so it is difficult to establish mathematical models of them, trajectory prediction is the best way to obtain the position of them. In order to aligning the bolt, a prediction algorithm of multi-sensor information fusion optimal Kalman filter is adopted to predict the trajectory of the camera which can improves the accuracy of the prediction. The trajectory of the bolt adopts a least squares method based on the interest area to meet the requirements for real time response of fast visual servo. Since the frictions in different screw threads are uncertain, merely improving the control precision of torque can't assure the conformity of the pre-tightening force, so torque coefficient control method is proposed to solve this problem.

\section{The BAsic StRUCtURE OF THE RoBOT}

\section{A. The Basic Structure of the Bolt Tightening Robot}

As shown in Fig. 1, the proposed bolt tightening robot consists of the main body, two walking wheels with corresponding locking clamps, equipotential wheels, two arms and the bolt tightening unit. The main body of the robot 
is equipped with a gyroscope and accelerometer to detect the angle.

\section{B. Improvements for Our Robot}

Aiming at the bolt tightening task on the power transmission lines, We designed a bolt tightening unit which consists of a mechanical arm with multi-degree-of-freedom (DOF)and the endmost bolt tightening performer for our robot. The multi-DOF arm has three joints which locate at the both ends and the center of the arm. By three joints' collaboratively working, the multi-DOF arm can take the endmost performer to anywhere we want.

The endmost tightening performer is made up of pipe spanners, close focusing cameras, micro-driving motors and several support mechanisms. The two close focusing cameras are installed at the right center of pipe spanners' tail end as shown in Fig. 2, once the pipe spanner is adjusted to zero in the bolt's center, the following propelling and rotating step can be implemented without calculating the visual deviations. During the operation of bolt tightening, one motor turns gears to rotate one of the pipe spanners and the other drives two pipe spanners propel towards each other or to the opposite direction, thus realizing the bolt tightening work.

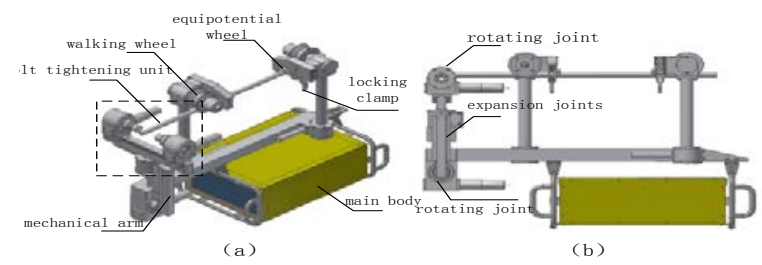

Figure 1. The basic structure of the bolt tightening robot

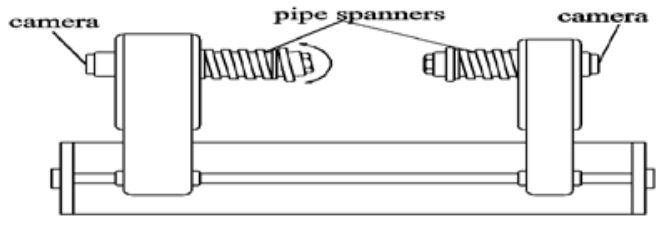

Figure 2. The endmost tightening performer

\section{The ViBration Prediction of Bolt}

In order to complete the alignment of bolt, the position of the camera and the bolt should be known. Considering the influence of the wind, the motion of the robot and the bolt are nonlinear, and their vibration frequency is different, so it is difficult to establish mathematical models of them, trajectory prediction is used to obtain their position.

\section{A. The Trajectory Prediction of Robot}

As the data from the accelerometer has the measurement noise, hence tilting measurement only using accelerometer is not effective enough to get accurate data. A gyroscope offers angular velocities around the three axes but it can produce the drift error. In short, taking the advantages and disadvantages of gyroscopes and accelerometers into consideration, the data fusion is an essential for a reliable tilt sensing especially for real time applications.

This paper proposes a type of multi-sensor information fusion Kalman filter prediction algorithm. To solve the problem of noise jamming and the attitude optimal estimation, the collected data is fusioned using the Kalman filter method.

\section{B. Data Fusion Using Kalman Filter}

Noises in measurement data contribute to the errors. In the data fusion process, the Kalman filter takes the noises into account via covariance matrices and updates the matrices at each time interval. It estimates the state of the system based on the current a nd previous states. The precision of the data filtered is significantly improved. Such a process based on the accelerometer model and gyroscope model.

The accelerometer model is represented as:

$$
\varphi_{\text {acc }}=\varphi_{\text {extra }}-\mathrm{g}+\mathrm{b}_{a}+\mathrm{n}_{a}
$$

Where, $\varphi_{\text {extra }}, g, \mathrm{~b}_{a}, \mathrm{n}_{a}$ means external acceleration, gravitational acceleration, accelerom eter bias and noise respectively.

A gyroscope is used to measure the angular velocity around three mutually perpendicular axes. It is not easy to be affected by external interference but its data will suffer from drifting in long term.

$$
\omega_{\mathrm{gyro}}=\omega+\mathrm{b}_{\mathrm{g}}+\mathrm{n}_{\mathrm{g}}
$$

Here, $b_{g}$ and $n_{g}$ are the gyroscope bias and noise. The Kalman filter operates by producing a statistically optimal estimation of the system state relied upon the measurements. The filtering process is based on the noise of the input to the filter called the measurement noise and the noise of the system itself called the process noise. To make things easier, assume the noises are Gaussian distributed and have a mathematical expectation of zero.

Equation (3) and (4) are the standard Kalman filter expressions.

$$
\begin{gathered}
x_{k}=F x_{k-1}+B u_{k-1}+w_{k} \\
z_{k}=H x_{k}+v_{k}
\end{gathered}
$$

$x_{k}$ is the system state matrix at time $k$, which is given by:

$$
x_{k}=\left[\begin{array}{c}
\dot{\varphi} \\
\dot{\mathrm{b}}
\end{array}\right]_{k}
$$

The outputs of the filter are the angle $\theta$ and the bias $\dot{\theta}_{\mathrm{b}}$ based upon the measurements from the accelerometer and 
gyroscope. The bias is the amount that the gyroscope has drifted. $\mathrm{F}$ is the state transition matrix which is applied to the previous state $x_{k-1}$. For the robot contains a three-axis accelerometer and a three-axis gyroscope, $\mathrm{F}$ is defined as:

$$
F=\left[\begin{array}{cc}
1 & -\triangle t \\
0 & 1
\end{array}\right]
$$

$u_{k}$ is the control input. For the data fusion discussed, it is the gyroscope measurement in degrees per second at time k, which is also called the angular rate $\dot{\theta}$. Then the state Equation (3) can be rewritten as:

$$
x_{k}=F x_{k-1}+B \omega_{k-1}+w_{k}
$$

$\mathrm{B}$ is called the control matrix and is defined by:

$$
B=\left[\begin{array}{c}
\Delta t \\
0
\end{array}\right]
$$

Angle $\theta$ is obtainable through Equation (8) when multiplying the rate $\dot{\theta}$ by time $\Delta t$, since the bias cannot be calculated directly based on the angular velocity the bottom of the matrix is set to zero.

In equation (3) and (4), the variables $w_{k}$ and $v_{k}$ represent the process and measurement noise respectively. They are assumed to be independent of each other and with normal probability distributions (Gaussian white noise) [4].

$$
\begin{aligned}
w_{k} & \sim N\left(\begin{array}{ll}
0 & Q_{k}
\end{array}\right) \\
v_{k} & \sim N\left(\begin{array}{ll}
0 & R
\end{array}\right)
\end{aligned}
$$

$Q_{k}$ is the process noise covariance matrix which represents the state estimation of the accelerometer and bias. If consider the estimation of the bias and the accelerometer to be independent, then $Q_{k}$ is equal to the variance of the estimation of the accelerometer and bias.

$$
Q_{k}=\left[\begin{array}{cc}
Q_{\varphi} & 0 \\
0 & Q_{b}
\end{array}\right]
$$

The $Q_{k}$ covariance matrix depends on the current time k. So the accelerometer variance $Q_{\theta}$ and the variance of the bias $Q_{\dot{\theta}_{b}}$ are multiplied by time $\Delta t$. As the time goes, the process noise will be increased since the last update of the state.

In equation (6), $Z_{k}$ is the measured output. $\mathrm{H}$ is the measurement matrix and is used to map the true state space into the observed space. The true state cannot be observed since the measurement is just from the accelerometer. $\mathrm{H}$ is given by:

$$
H=\left[\begin{array}{ll}
1 & 0
\end{array}\right]
$$

The measurement noise covariance $\mathrm{R}$ is not a matrix. It is equal to the variance of the measurement noise since the covariance of the same variable is equal to its variance.

$$
R=\left[\begin{array}{ll}
v_{k} & v_{k}^{T}
\end{array}\right]=\operatorname{var}\left(v_{k}\right)
$$

Assume that the measurement noise is the same and does not depend on the time $\mathrm{k}$ :

$$
\operatorname{var}\left(v_{k}\right)=\operatorname{var}(v)
$$

If the measurement noise variance $\operatorname{var}(v)$ is set too high, the filter will respond slowly as it trusts new measurements less. On the contrast, if the value is set too low the filter will overshoot and be noisy since it trusts the accelerometer measurement too much. Therefore, the Kalman filter can be written as:

$$
\left\{\begin{array}{c}
{\left[\begin{array}{c}
\dot{\varphi} \\
\dot{b}
\end{array}\right]=\left[\begin{array}{cc}
1 & -1 \\
0 & 1
\end{array}\right]\left[\begin{array}{l}
\varphi \\
b
\end{array}\right]+\left[\begin{array}{l}
1 \\
0
\end{array}\right] \omega_{\text {gyro }}+\left[\begin{array}{c}
\omega_{g} \\
o
\end{array}\right]} \\
\varphi_{\text {acce }}=\left[\begin{array}{ll}
1 & 0
\end{array}\right]\left[\begin{array}{l}
\varphi \\
b
\end{array}\right]+\omega_{a}
\end{array}\right.
$$

The discrete system is made $\Delta t$ for the system sampling period, the state equation and measurement equation:

$$
\left\{\begin{array}{c}
x(k)=\left[\begin{array}{cc}
1 & -\Delta t \\
0 & 1
\end{array}\right] x(k-1)+\left[\begin{array}{c}
\Delta \mathrm{t} \\
0
\end{array}\right] \omega_{g y r o}(k-1) \\
+\left[\begin{array}{c}
\omega_{g}(k) \Delta t \\
o
\end{array}\right] \\
\varphi_{\text {acce }}=\left[\begin{array}{ll}
1 & 0
\end{array}\right] x(k)+\omega_{a}(k)
\end{array}\right.
$$

The implementation of Kalman filter includes two main steps: predict process and update process [5].

In predict process, the filter will first estimate the current state and the error covariance matrix at time $\mathrm{k}$. The next step for the filter is to estimate the priori error covariance matrix $P(k / k-1)$ based on the previous error covariance matrix $P(k-1 / k-1)$, which is defined by:

$$
P(k / k-1)=A P(k-1 / k-1) A^{T}+Q
$$

The matrix $P(k / k-1)$ is used to estimate how much trust can be put on the current values of the estimated state. The 
smaller the values are, the more trust on the current estimated state.

In the update process, the filter first computes the difference between the measurement $\mathrm{z}_{\mathrm{k}}$ and the priori state $\hat{x}(k-1 / k-1)$.Then the filter calculates the innovation covariance. The Kalman gain is therefore defined by:

$$
K_{g}(k)=P(k / k-1) H^{T} /\left[H P(k / k-1) H^{T}+R\right]
$$

Updating the posteriori estimation of the current state gives. Finally update the posteriori error covariance matrix by:

$$
P(k / k)=\left[I-K_{g}(k) H\right] P(k / k-1)
$$

\section{The Trajectory Prediction of Bolt}

A least square method based on the particular range is proposed to predict the trajectory of the bolt. According to the historical data, the trajectory of the bolt can be fitted out. On account of the historical forecasting precision, the weighted method is adopted to decide the size of the search area. A group of known historical position data is $\left(x_{i}, z_{i}\right) \quad(i=1,2,3 \ldots \ldots, m)$, and description of target trajectory curve is $P_{n}(x)$, make the sum of the squares of fitting error minimum [6],

$$
\min I=\sum_{i=1}^{m} r^{2}=\sum_{i=1}^{m}\left(p_{n}\left(x_{i}\right)-z_{i}\right)^{2}
$$

In the above (10), $i=1,2,3 \ldots . . . m, \quad r_{i}=p_{n}\left(x_{i}\right)-y_{i}$, $p_{n}(x)=\sum_{i=1}^{m} a_{k} x_{k}(n \leq m) \quad$ and $\quad a_{k}(k=0,1, \ldots \ldots, n) \quad$ is $\quad$ the constant coefficient of $p_{n}(x)$.From the multivariate extreme value calculation can obtain:

$$
\begin{gathered}
\frac{\partial I}{\partial a_{j}}=2 \sum_{i=1}^{m}\left(\sum_{k=0}^{n} a_{k} x_{i}^{k}-y_{i}\right) x_{i}^{j}=0, j=0,1 \ldots, n \\
\sum_{k=0}^{n}\left(\sum_{i=1}^{m} x_{i}^{j+k}\right) a_{k}=\sum_{i=1}^{m} x_{i}^{j} y_{i}, j=0,1, \ldots, n
\end{gathered}
$$

According to the type we can solve $a_{0}, a_{1}, a_{2}, \ldots \ldots ., a_{m}$ which can describe the trajectory curve $P_{n}(x)$ of the bolt.

In a certain region of a plane, position of the bolt is $\left[X_{k}, Z_{k}\right]$ in the $K T$ moment, $T$ is the sampling period. Firstly, consider the prediction of $X$ direction, according to historical data $[(k-m+1) T, x(k-m+1)]$ $[(k-m+2) T, x(k-m+2)], \ldots . . .,[k T, x k]$, using the least squares fitting out $X$ direction of trajectory $x=P_{n}(t), n$ is the times of polynomial $P$.According to the fitting polynomial, predicted position in $X$ direction of bolt is $x_{f}(k+1)$ at $(k+1) T$ moment. Prediction of $Z$ direction is same to $X$ direction.

There must exist error between the actual position and the predict position which influence by fitting precision of the least square method. In addition, the bolt has its own size, so the predicted position of bolt as the center, search range related to the above items. The weighted method is adopted to decide the size of search area. As shown in Fig. 4, image processing and searching in this range:

$$
\begin{aligned}
& D_{x}=\sum_{p=0}^{m-1} b(m-p)\left|x_{f}(k-p)-x(k-p)\right| \\
& D_{z}=\sum_{p=0}^{m-1} b(m-p)\left|z_{f}(k-p)-z(k-p)\right|
\end{aligned}
$$

Here, $D_{x}$ and $D_{z}$ are respectively the width and height radius of search area, $m$ is the number of reference position, $b$ is constant. Type (13) and (14) $\mathrm{m}$ - $\mathrm{P}$ item is all of the weighted value of the prediction error, the size of the interest area is directly related to historical accuracy.

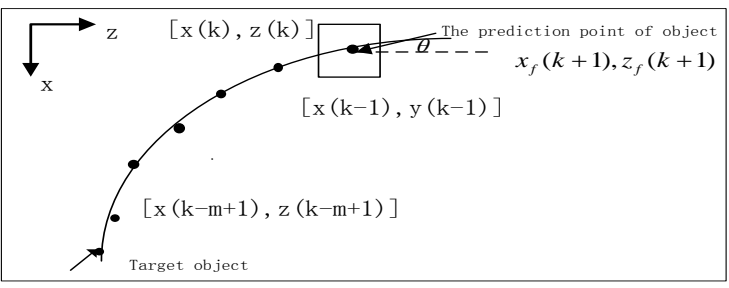

Figure 3. Curve fitting and image search area

\section{TORQUE COEFFICIENT CONTROL}

To improve the fatigue life, realiablity and quality of screw joint, the key point is to control the precision of pretightening force. Pre-tightening force overabundance result in serious consequences such as connection part of bolt be crushed, distorted or fractured; Pre-tightening force is insufficient cause nut loose[7]. Nowadays, the commonly used method of tightening bolt is torque control method, which causes decentralization of axial pre-tightening force, and decrease the reliability of the joint. Other methods are not suitable for the miniature screw thread precise assembly which need target pre-tightening force determined, since the control force is too large.

\section{A. The Principle of Torque Coefficient Control}

After the nut appressed the gasket, the relationship between pre-tightening force and angle is linear, pretightening force on the nut is:

$$
F=\frac{C_{S} P \theta}{2 \pi}
$$


In the type, $F$ is prestressing force; $C_{S}$ is total stiffness of thread vice; $P$ is the thread pitch; $\theta$ is nut corner. Nut angle $\theta$ is proportional to the motor speed $\omega$ varies with time $t$ :

$$
\theta=\omega \cdot t
$$

The relationship between thread vice tightening torque $\mathrm{T}$ and pre-tightening force $\mathrm{F}$ in process of tightening is:

$$
T=F\left[\frac{d_{2}}{2} \tan \left(\phi+\varphi_{V}\right)+\mu d_{\omega}\right]
$$

In the type, $d_{2}$ is the thread pitch diameter; $d_{\omega}$ is bearing surface equivalent diameter. $\mu$ is the friction factor between the nut and bearing surface; $\phi$ is the thread angle; $\varphi_{V}$ is the equivalent friction angle. The torque coefficient is: $k=\frac{d_{2}}{2 d} \tan \left(\phi+\varphi_{V}\right)+\mu \frac{d_{\omega}}{d}, \quad d$ is threaded nominal diameter.

In type (25), after $\mathrm{k}$ instead of each influence factor, we can find

$$
T=k F d
$$

The slope $K_{\mathrm{t}}=\mathrm{d} T / \mathrm{dt}$, from type (25) to type (27), we can find,

$$
K_{\mathrm{t}}=\frac{k d \mathrm{P} \omega}{2 \pi}\left(C_{\mathrm{s}}+\mathrm{t} \frac{\mathrm{d} C_{\mathrm{s}}}{\mathrm{dt}}\right)
$$

If the system stiffness is known, $\frac{\mathrm{d} C_{\mathrm{s}}}{\mathrm{dt}}=0$. The equivalent stiffness $C_{t}=C_{s}+t \frac{\mathrm{dC}_{\mathrm{s}}}{\mathrm{dt}}=C_{s}$, so

$$
F=\frac{C_{S}}{K_{t}} \frac{P \omega}{2 \pi} T
$$

$K_{\mathrm{t}}$ and $C_{\mathrm{s}}$ is the core of the torque coefficient. If the system stiffness is known, $C_{S}=F / \delta, F$ is the force on the bolt, $\delta$ is the deformation of the bolt component, according to the torque slope change over time, control torque can be calculated from the corresponding pretightening force. When the different thread vice assembly, friction coefficient $\mu$ change causes the change of $K_{\mathrm{t}}$, so the different control torque can be calculated to ensure the consistency of the pre-tightening force.

\section{B. The Measurement of $K_{\mathrm{t}}$}

This paper adopts the high precision of torque sensor to measurement torque of bolt. In the process of assembly thread pair, there are three stages of torque changes over time:(1)before the component appressed the nut is the nonlinear increased stage; (2) appressed the nut is the linear increased stage;(3)after reach the control torque is the stage of torque plunged and maintenance. As shown in Fig. 4.Pretightening force is the same. As shown in Fig. 5. When the torque entering the linear increase stage, in theory $K_{\mathrm{t}}$ is a constant value, when actual measurement, because torque has slight fluctuation, in the practical calculating $K_{\mathrm{t}}$ need choose torque values in a set period of $\Delta \mathrm{t}$ time, and use the least squares method to fitting the curve, make the slope of the fitting line is $K_{t}$, When $\Delta t$ is long enough, the torque fluctuation influence on $K_{\mathrm{t}}$ will be significantly reduced, using this method can calculate the curve of the $K_{t}$ changes over time, when the torque change into the linear trend, $K_{\mathrm{t}}$ start to leveled off, as shown in Fig. 6. When $\mathrm{t}=1.8 \mathrm{~s}$, choose the $K_{\mathrm{t}}$ to calculate the control torque.

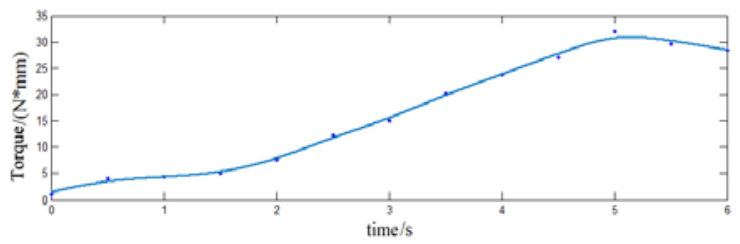

Figure 4. The torque curve over time

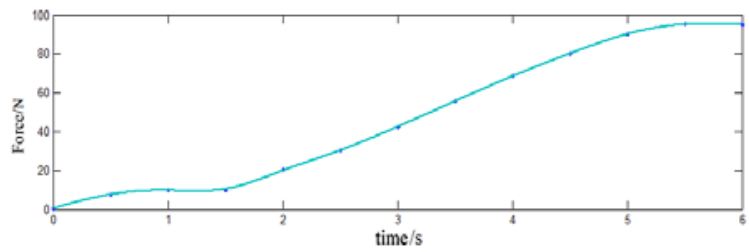

Figure 5. The curve of pre-tightening force

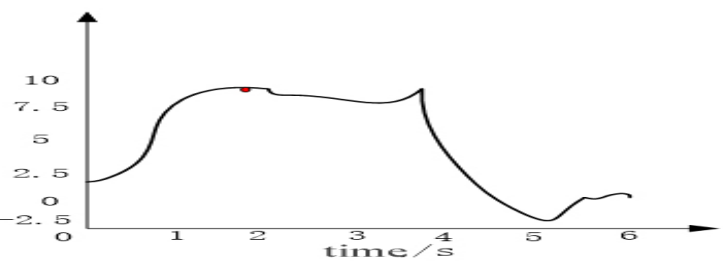

Figure 6. The curve of the $K_{t}$ changes over time

\section{System Stiffness of Judgment}

Due to the motor do uniform motions, the deformation of thread pairs and other change linear over time. Because system containing force sensors, when bolt under the pretightening force, the deformation of thread pair and other is far smaller than deformation of the force sensor, so the stiffness of the force sensor can be defined as the system stiffness. As shown in Fig. 7 can confirm force sensor is the constant stiffness system. So the system stiffness can be calculated from the figure. 


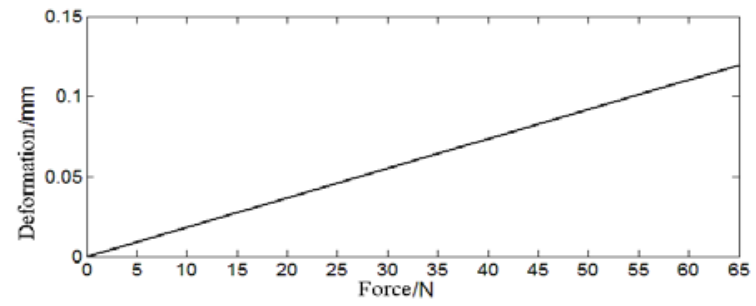

Figure 7. The curve of the $K_{t}$ changes over time

\section{EXPERIMENT RESULTS}

\section{A. The Kalman Filter Fusion Result}

In order to verify the validity of the Kalman filtering sensor fusion method, build verification system is as follow:

TABLE I. SYSTEM EXPERIMENT PARAMETERS

\begin{tabular}{|c|c|c|}
\hline Microcontroller & Gyroscope & Accelerometer \\
\hline TMS320F28335 & IMU610 & SCA3000 \\
\hline
\end{tabular}

TABLE II. The Initialization Parameter of FilteR

\begin{tabular}{|c|c|c|c|c|c|c|}
\hline $\begin{array}{c}\text { Sampling } \\
\text { Period }\end{array}$ & $\mathrm{A}$ & $\mathrm{B}$ & $\mathrm{Q}$ & $\mathrm{R}$ & $X_{0}$ & $P_{0}$ \\
\hline $20 \mathrm{~ms}$ & {$\left[\begin{array}{cc}1 & -0.02 \\
0 & 1\end{array}\right]$} & {$\left[\begin{array}{c}10.02 \\
0\end{array}\right]$} & {$\left[\begin{array}{cc}0.01 & 0 \\
0 & 0.005\end{array}\right]$} & {$\left[\begin{array}{l}0.056\end{array}\right]$} & {$\left[\begin{array}{c}\mathbf{O} \\
\mathbf{O}\end{array}\right]\left[\begin{array}{ll}0.005 & 0.005 \\
0.005 & 0.005\end{array}\right]$} \\
\hline
\end{tabular}

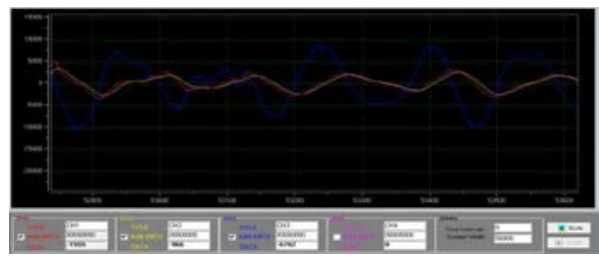

Figure 8. Waveform curve after introduces the Kalman filter

In Fig. 8, the red line represents the gyroscope; the blue line represents the accelerometer. The yellow line represents the curve after Kalman filter data fusion. Fig. 8 is the waveform curve after introduces the Kalman filter, the accelerometer succeeded in tracing out the deviation of the gyroscope, the output curve of filter is smoothing, the result of the filter is obviously, the accuracy of angle estimation is greatly improved.

\section{B. A Least Squares Method Based on the Interest Area}

Using this new type of least-square method, we can get $X$, $\mathrm{Z}$ axis curve changes over time. Fig. 9 represents when use new type of least square method, the actual measurement curve and forecasting curve of bolt on $\mathrm{X}, \mathrm{Z}$ axis direction relative to the target location. Fig. 10 is the moving trajectory of bolt under real-time object tracking based on prediction. Because this paper only consider the slightly wind, so its trajectory is a very smooth roll movement.

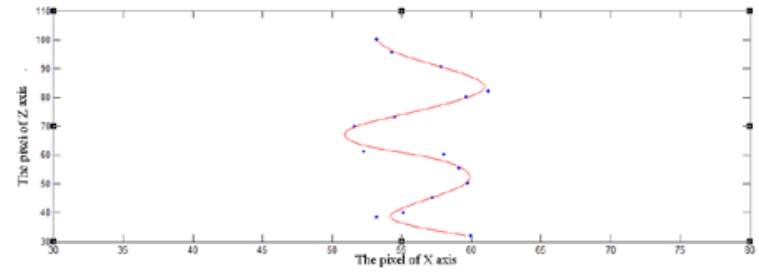

Figure 9. Moving trajectory of bolt under real-time object tracking

Table III is respectively using least square method, the article [8] particle filter method and the article [9] the $\alpha-\beta$ filtering method to predict the position of bolt. From the table we can get the least-square method use the shortest time to calculating.

TABLE III. PERFormance OF DifFERENT PREDICTION AlgorithmS

\begin{tabular}{|c|c|c|c|}
\hline Method & $\begin{array}{c}\text { The Method of } \\
\text { This Paper }\end{array}$ & $\alpha-\beta$ Filter & Particle Filter \\
\hline $\begin{array}{c}\text { Computation } \\
\text { Time/ms }\end{array}$ & 3.11 & 38.14 & 131.45 \\
\hline
\end{tabular}

Fig. 10 respectively represent $1,8,16,22,28,34,42$ frame of the bolt images, the red point represent the prediction position at that moment, green box is the search area. It can be indicated from the picture that the searching area is reduced into a relatively small one after the prediction; even the biggest area is not more than one fifth of the total moving region, thus assuring the rapidity of the object's information extraction.

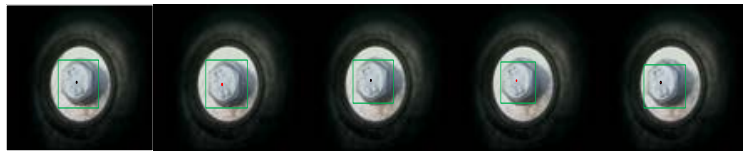

Figure 10. Images of bolt under real-time object tracking based on prediction

\section{Experiment of Torque Coefficient Control Method}

Using ten group of thread vice assembly to conduct experiment, set the pre-tightening force respectively100N,130N,160N,the measured result of pretightening force is shown in Fig. 11, the corresponding torque control is shown in Fig. 12. The control precision of pretightening force is respectively $\pm 5.5 \%, \pm 5.4 \% \pm 4.3 \%$, the corresponding torque discrete degrees is $\pm 19.8 \%, \pm 20.6 \%$, $\pm 29.9 \%$, which can prove that torque coefficient control method can achieve good control precision of pre-tightening force, when the torque control discrete degree is bigger, the pre-tightening force still can obtained smaller discrete degree.

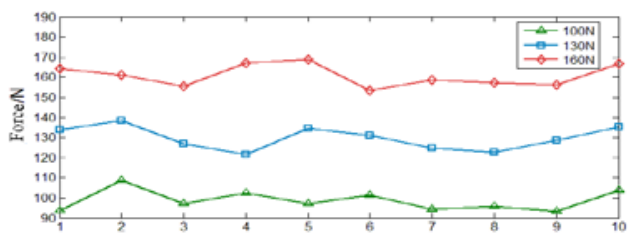

Figure 11. The actual value of pre-tightening force 


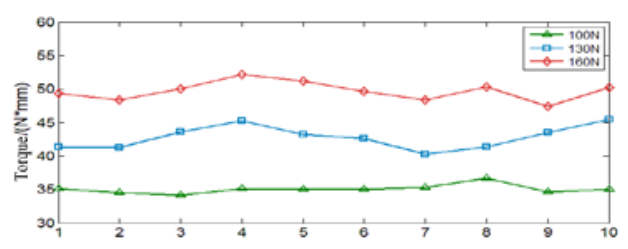

Figure 12. The corresponding control torque

As shown in Fig. 13, 14, is the result of the torque control method. Torque tightening method is determined by the relationship between the pre-tightening force $(T)$ and the tightening torque $(F) . T=k F d, d$ is the thread nominal diameter, $k$ is the torque coefficient. This method only control a certain torque. In experience design, torque coefficient value generally take 0.2 .The control precision of pre-tightening force is respectively $\pm 8.3 \%, \pm 12.7 \%$, $\pm 9.5 \%$. The corresponding torque discrete degrees is $\pm 2.1 \%, \pm 3.2 \% \pm 2.6$.Compare with our method, it can clearly see that our method is more effectively and have high precision.

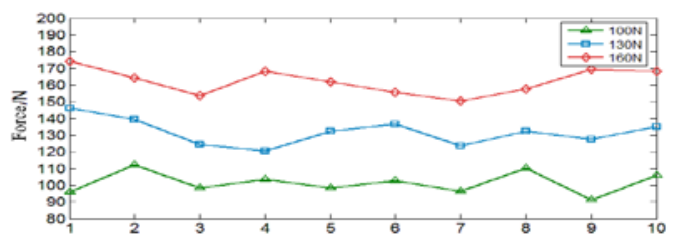

Figure 13. The actual value of pre-tightening force

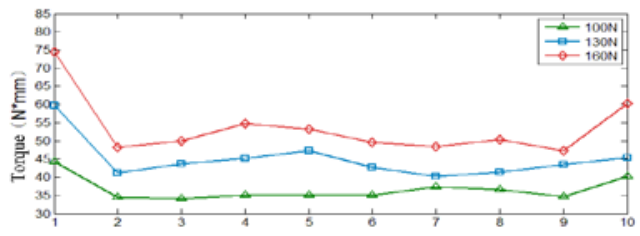

Figure 14. The corresponding control torque

\section{The Result of the Infrared Detector}

As shown in Fig. 15, when the bolt looseness, it will change the heat conduction of the object, the surface temperature of bolt will change. And the surface temperature could become a clear contrast with the outside temperature that can determine whether the bolt looseness. The last picture show that the bolt after the tightening strategy, it can be better tightened.

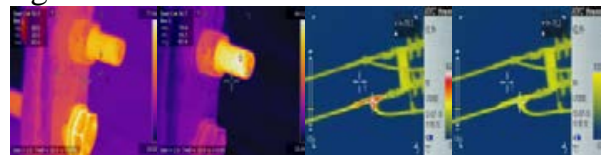

Figure 15. The result of infrared detector

\section{CONCLUSION}

This paper discusses the process of bolt alignment. When mechanical arm is under the disturbance of wind, how to achieve the track prediction of them. This paper adopts a multi-sensor Kalman filter algorithm which can improve the accuracy of the camera location prediction. Bolt location prediction is use method of least squares based on interest area which can satisfy real time of the system. The paper adopts the infrared detector to detect loose bolts; torque coefficient control method is used to control the torque. Experimental results show that the proposed bolt aligning and tightening strategy can achieve the work timely and have high efficiency. It can achieve a visual servo control method of bolt tightening robot on power transmission lines.

\section{ACKNOWLEDGMENT}

This work was supported by the National Natural Science Foundation of China (Grant No. 61473049).

\section{REFERENCES}

[1] G.P.Luo,J.G.Lu and J.G. Zhang,"Failure analysis on bolt-type power connector's application,”Electrical Contacts,1999. Proceedings of the Forty-Fifth IEEE Holm Conference on, Pittsburgh, PA, USA, 1999, pp. 77-86.

[2] E.Tani,H.Yamada,R.Kato,K.Kurabe, K.Yamashita and K. Tatsuno,"Development of the tightening nut task skill using a power distribution line maintenance experimental robot,"2015 IEEE/SICE International Symposium on System Integration (SII), Nagoya, 2015, pp. 558-563.

[3] Chu B,Jung K, Kang H K, et al.Mechanism and analysis of a robotic bolting device for steel beam assembly[C]// Control Automation and Systems (ICCAS), 2010 International Conference on. IEEE, 2010:2351-2356.

[4] Tsukahara K, Tanaka Y, He Y, et al. An experimental robot system for power distribution line maintenance robots - System architecture and bolt insertion experiment -[C]// Ieee/rsj International Conference on Intelligent Robots and Systems. IEEE, 2008:1730 - 1736.

[5] Gui P, Tang L, Mukhopadhyay S. MEMS based IMU for tilting measurement: Comparison of complementary and kalman filter based data fusion[C]// Industrial Electronics and Applications. IEEE, 2015.

[6] Tang G, Qiu Y. Improved least square method apply in ship performance analysis [J]. 2010, 5:V5-594-V5-596.[6] Gong Junliang. Research on real-time infrared small targets detection and tracking[D].Changchun Institute of Optics,Fine Mechanics and Physics Chinese Academy of Sciences ,May,2013.

[7] You X,Li B,Chen W, et al.Tension distribution algorithm of a 7-DOF cable-driven robotic arm based on dynamic minimum pre-tightening force[C]// IEEE International Conference on Robotics and Biomimetics.2011:715-720.

[8] Song Sha, Wang Xiaodong, Luo Yi.Precise Control of Pre-tightening Force for Miniature Screw Thread Joints[J].China Mechanical Engineering, 2010(13):1523-1527.

[9] Li Pengfei, Yu Jianping, Li Liangqun. Maneuvering target tracking based on fuzzy adaptive $\alpha-\beta$ filter [J]. Systems Engi-neering and Electronics, 2008, 30(11): 2138-2141. 